\title{
An Improved Method of the Energy Loss Calculation Considering the Volatility of Wind Power Generation
}

\author{
Bo Ruan1, Tingting Hou1, Yu Li² ${ }^{2 *}$ Zhen Mei², Jun Huang2 \\ ${ }^{1}$ Research Institute of Economics and Technology, Hubei Electric Power Company Limited, Wuhan, China \\ ${ }^{2}$ State Key Laboratory of Advanced Electromagnetic Engineering and Technology, Huazhong University of Science \& Technology, \\ Wuhan, China \\ Email:969180944@qq.com, 277921920@qq.com
}

How to cite this paper: Ruan, B., Hou, T.T., Li, Y., Mei, Z. and Huang, J. (2017) An Improved Method of the Energy Loss Calculation Considering the Volatility of Wind Power Generation. Energy and Power Engineering, 9, 281-291.

https://doi.org/10.4236/epe.2017.94B033

Received: February 20, 2017

Accepted: March 30, 2017

Published: April 6, 2017

\begin{abstract}
The energy loss of the power grid is one of the key factors affecting the economic operation of power systems. How to calculate the electric energy consumption accurately will have a great influence on the planning, operation and management of the power grid. Currently there is a mountain of theoretical methods to calculate the line loss of the power system. However, these methods have some limitation, such as less considering the volatility of wind power resources. This paper presents an improved method to calculate the energy loss of wind power generation, considering the fluctuations of wind power generation. First, data are collected to obtain the curve of the typical daily expected output of wind farms for one month. Second, the curve of the typical daily expected output are corrected by the average electricity and the shape factor to obtain the curve of the typical daily equivalent output of wind farms for one month. Finally, the power flow is calculated by using typical daily equivalent output curve to describe the energy loss for one month. The results in the $110 \mathrm{kV}$ main network show that the method is feasible.
\end{abstract}

\section{Keywords}

The Energy Loss of the Power Grid, Wind Power Generation, Shape Factor, the Curve of the Typical Daily Equivalent Output, Power Flow Calculation

\section{Introduction}

The power loss analysis is a method to acquire the power loss degree in the process of power supply, and one of the important economic and technical indicators to assess the power supply business, which provides a theoretical basis for 
future reduction of power loss [1] [2] [3]. Hence, the economical and practical significances of power loss analysis cannot be underestimated.

As the advantage of environmental friendly and sustainable development capability, the renewables increase rapidly. Renewable energy is considered to be one of the effective measures to achieve energy saving, and can replace traditional power. Compared with conventional power sources, wind power, photovoltaic energy and other renewable energy sources have features of randomness, volatility, regionalism and so on [4] [5] [6]. Some researches show that wind farm output has very large volatility both for days and years. Figure 1 shows the output of a wind farm for each 15 min intervals for two consecutive days:

As shown in Figure 1, the curve of the wind farm output for two consecutive days are quite different. The previous studies usually treat wind power the same as the conventional resources, which consider the one hour output is basically constant and ignoring the volatility of output in any selected typical days. It can be seen from the definition of the energy loss, which is obtained from the integral of the power loss over a period of the time. As if the fluctuation of the wind power output is not considered, the power loss error will occur in the calculation.

Zhang mentions that wind power generation is considered as "nondisposable" due to the randomness and intermittence [7]. Wu introduces the equivalent power method to calculate the energy loss of wind power integration [8]. Shape factor $\mathrm{K}$ is defined in [9] [10], which can be used to correct the average to reflect the volatility of a curve over a period of the time.

This paper presents an improved method to calculate the energy loss of wind power generation, with consideration of the random fluctuations of wind power. In order to describe the volatility of wind farms, the energy loss can be calculated

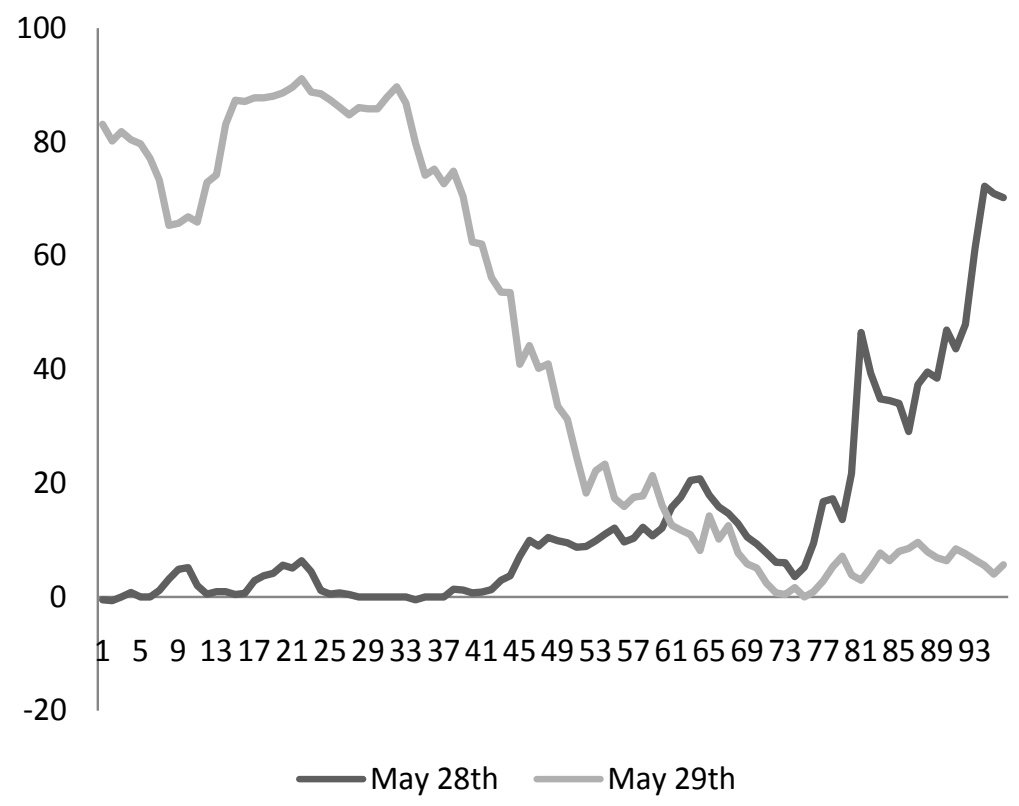

Figure 1. The output of a wind farm for each 15 min intervals for two consecutive days. 
more accurately making use of the shape factor. The validity of proposed method is verified by a case of $110 \mathrm{kV}$ main networks.

\section{The Original Method of Energy Loss Calculation}

\subsection{The Typical Daily Method of Energy Loss Calculation}

The energy loss is divided into two parts, one is caused by transformers, lines, reactors, capacitors and so on, and the other contains corona loss and station power loss, which are considered as constants in the calculation. Therefore we focus on the energy loss calculation in the power system caused by transformers, lines, reactors, capacitors and so on in this paper. When calculating the monthly energy loss, the power flows repeatedly suffer from the heavy computational burden, so the basic idea is to calculate the energy loss of the typical day, and estimate the monthly energy loss [11].

In the original method of energy loss calculation, the outputs of the load and the generation for each time period is considered as constants. The energy loss of the typical day can be calculated as shown in Equation (1):

$$
\Delta A_{d}=\left[\sum_{l=1}^{L}\left(3 \sum_{t=1}^{24} I_{t, l}^{2} R_{t, l}\right)+\sum_{m=1}^{M} \sum_{t=1}^{24} V_{t, m}^{2} G_{t, m}\right] \times 10^{-3}=\Delta A_{R}+\Delta A_{G}(\mathrm{kWh})
$$

Here, $\Delta A_{d}$ is the energy loss of the typical day $(\mathrm{kWh}), I_{t, l}$ is the current of branch $I$ at time $t(A), R_{t, l}$ is the resistance of branch $I$ at time $t(\Omega), \mathrm{L}$ is the number of power lines and transformer branches; $V_{t, m}$ is the voltage of node $\mathrm{m}$ at time $t(V), G_{t, m}$ is the transformer core loss or equivalent conductance of shunt reactors and capacitors of node $\mathrm{m}(\mathrm{S}), \mathrm{M}$ is the number of grid transformers.

Since $\Delta A_{G}$ is considered as a constant in the calculation, this paper focuses on studying line loss $\Delta A_{R}$.

$I_{t, l}, V_{t, m}$ and other operating parameters are obtained from the power flow.

\subsection{A Method for Correcting the Typical Daily Curve}

Based on the information of active and reactive power injections of the generators and loads, the power flow can be calculated to acquire the outputs of the voltage/current of each nodes and the power of each branches.

In the calculation of line loss, watt-hour meters are considered more accurate than power meters. If both meters are accurate, the integral value of daily power should be consistent with the value of watt-hour meters for each day. Otherwise, the power for each hour will be corrected by the average electricity according to the curves of the load and the generation. The process of the method is shown as follows: is: first, the curves of the load and the generation for each bus are converted into the table of the load factor and the generation factor. Then, the average power is converted by daily electricity. Finally, the 24-hour data of the load and the generation of every node are formed for power flow calculation by multiplying the average power by the corresponding table of the load factor and the generation factor [12] [13]. 
The active load factor for node $i$ at time $t$ is shown in Equation (2):

$$
f_{i, t}=\frac{P_{i, t}}{P_{a v i}}
$$

$P_{a v i}$ is calculated by Equation (3):

$$
P_{a v i}=\frac{A_{i p}}{24 \times T_{m}} \times 10
$$

Here, $f_{i, t}$ is the active load factor of node $i$ at time $t, P_{i}$ is the measured power of node $i$ at time $t(\mathrm{MW}), P_{a v i}$ is the daily average active power of node $I$ (MW), $A_{i p}$ is the monthly active power supply of node $i(\mathrm{kWh}), T_{m}$ is the number of days in that month.

Suppose there are $H$ nodes in the grid, and the table of the load factor is presented in Equation (4):

$$
F_{P}=\left[\begin{array}{cccc}
f_{1,1} & f_{1,2} & \cdots & f_{1,24} \\
f_{2,1} & f_{2,2} & \cdots & f_{2,24} \\
\cdot & \cdot & \cdot & \cdot \\
\cdot & \cdot & \cdot & \cdot \\
\cdot & \cdot & \cdot & \cdot \\
f_{H, 1} & f_{H, 2} & \cdots & f_{H, 24}
\end{array}\right]
$$

24-hour load data can be acquired by typical daily power supply of every node.

$$
P_{i, t}=\frac{A_{i, P}}{24} \times f_{i, t} \times 10(\mathrm{MW})
$$

Reactive power can be acquired in the same way. The generator node can be acquired as well as the load node.

In summary, the corrected method can make up for the error caused by power meters, and fully take into account the influence of the curve of the load and the generation to the line loss.

\subsection{The Method of Estimating the Monthly Energy Loss}

The estimation of the monthly energy loss is shown in Equation (6):

$$
\Delta A_{m}=\Delta A_{d} \times T_{m}
$$

Here, $\Delta A_{m}$ is the monthly line loss $(\mathrm{kWh}), \Delta A_{d}$ is the line loss of any typical days $(\mathrm{kWh})$.

\section{The Method of the Energy Loss Calculation Considering the Volatility of Wind Power Generation}

\subsection{The Curve of the Typical Daily Expected Output of Wind Farms}

From the above analysis, the volatility of wind power generation is too large to select a typical day. Therefore, the original method will cause a very large error. Based on the output characteristics of wind farms, this paper presents a method 
to acquire the curve of the typical daily expected output of wind farms for one month. The method is to collect data of the wind power output per hour per day of the whole month, and the superposition of the data per hour is divided by the number of days to acquire the expectation of wind power output per hour for that month. This method can get the curve of the typical daily expected output of wind farms for one month.

The expectation is shown in Equation (7):

$$
P_{m, t}==\frac{\sum_{k=1}^{T_{m}} P_{k, t}}{T_{m}}(\mathrm{MW})
$$

Here, $P_{m, t}$ is the active power expectation of the wind power output in hour $t(\mathrm{MW}), P_{k, t}$ is the active power expectation of the wind power output in hour $t$ on day $k(\mathrm{MW})$.

We replace the original curve of wind power output to the curve of the typical daily expected output of wind farms, and then correct it by the average electricity to calculate the power flow, and get the energy loss of a typical day via the power flow calculation, and estimate the monthly energy loss at last.

\subsection{The Curve of the Typical Daily Equivalent Output of Wind Farms}

The method in 3.1 still exists some problems. For example, the data of sampling points are too small to reflect the volatility of the wind power generation. The wind power output for each hour is considered as constants both in the original method and the method in 3.1, which is not consistent with the fact that the wind power output is fluctuant violently in every hour. Therefore, the method needs to be further improved.

With the improvement of the power grid dispatching automation level, most of the regional power grids have SCADA function, corresponding with the ability to collect more power grid operating parameters. To maximize the influence of the volatility of wind power generation to the line loss calculation, one sample point per hour is increased to $\mathrm{N}$ samples per hour in this section.

The energy loss at time $\mathrm{T}$ for each line is shown in Equation (8):

$$
\Delta A=3 \int_{0}^{T} I^{2} R \times 10^{-3} \mathrm{~d} t=3 I_{e q}^{2} R T \times 10^{-3}=\frac{P_{e q}^{2}+Q_{e q}^{2}}{V^{2}} R T \times 10^{-3}
$$

Here, $I_{e q}, P_{e q}$ and $Q_{e q}$ are equivalent values of the current, active power and reactive power [1].

$$
I_{e q}=\sqrt{\frac{1}{T} \int_{0}^{T} I^{2} \mathrm{~d} t}
$$

When the grid voltage is constant, $P_{e q}$ and $Q_{e q}$ also have the same expression as Equation (9). Thus, the so-called equivalent value is actually a RMS value.

Equivalent values of the current, active power and reactive power can be represented by their mean values. 


$$
\begin{aligned}
I_{e q} & =G I_{a v} \\
P_{e q} & =K P_{a v} \\
Q_{e q} & =L Q_{a v}
\end{aligned}
$$

After bringing in the average load, the energy loss formula can be rewritten as Equation (11):

$$
\begin{aligned}
\Delta A=3 G^{2} I_{\mathrm{av}}^{2} R T \times 10^{-3} & =\frac{R T}{V^{2}}\left(K^{2} P_{a v}^{2}+L^{2} Q_{a v}^{2}\right) \times 10^{-3} \\
P_{a v} & =\frac{A_{P}}{T} \\
Q_{a v} & =\frac{A_{Q}}{T}
\end{aligned}
$$

In this paper, we introduce the concept of the shape factor to the curve of the typical daily expected output of wind farms. We set $T$ to be an hour, collect the output data of the wind farm every day of the month, and calculate the mean and RMS values.

The time interval is set to be each one hour. So Equation (7) is corrected to Equation (13):

$$
P_{a v e, t}=\frac{\sum_{k=1}^{T_{m}} \sum_{n=1}^{N} P_{k, n, t}}{T_{m} \times N}(\mathrm{MW})
$$

The formula for the RMS value of $t$ period is as follows:

$$
P_{e q, t}=\sqrt{\frac{\sum_{k=1}^{T_{m}} \sum_{n=1}^{N} P_{k, n, t}^{2}}{T_{m} \times N}}(\mathrm{MW})
$$

Based on the definition, the shape factor is as follows:

$$
K_{t}=\frac{P_{e q, t}}{P_{\text {ave }, t}}=\sqrt{\frac{\sum_{k=1}^{T_{m}} \sum_{n=1}^{N} P_{k, n, t}^{2}}{T_{m} \times N}} / \frac{\sum_{k=1}^{T_{m}} \sum_{n=1}^{N} P_{k, n, t}}{T_{m} \times N}
$$

Here, $P_{\text {ave, }}$ is the mean value of active power of wind farms during the period $t(\mathrm{MW}), P_{e q, t}$ is the RMS value of active power of wind farms at period $t(\mathrm{MW})$, $P_{k, n, t}$ is active power of wind farms at $N$ sampling points during the period $t(\mathrm{MW}), N$ is the number of sampling points per hour, and $K_{t}$ is the shape factor of wind farms for the period $t$.

The curve of the typical daily equivalent output of wind farms for one month can be acquired by the corrected curve of the typical daily expected output using the method of 2.2. In the improved method, we replace the original curve of wind power output to the curve of the typical daily equivalent output of wind farms, and get the energy loss of a typical day via the power flow calculation, and estimate the monthly energy loss.

It should be pointed out that the improved method is also suitable for the case of conventional units and load nodes. 


\subsection{The Improved Energy Loss Calculation Procedures}

From 2.1 and 2.2 the improved energy loss calculation procedures are shown as follows:

1) Collect data to acquire the curve of the typical daily expected output of wind farms for one month and the expectation table of shape factors;

2) Correct the curve of the typical daily expected output of wind farms and other curves of the load and the generation by the corresponding average electricity;

3) Get the curve of the typical daily equivalent output of wind farms for one month by multiplying curve of the typical daily expected output by the corresponding shape factor;

4) Get the energy loss of a typical day via power flow calculation, and estimate the monthly energy loss of the power system.

\section{Case Study}

The structure of a $110 \mathrm{kV}$ main network is shown in Figure 2. There are two power generations. One is named Longkou, which is a conventional power generation; the other is named Xiangyang, which is a wind farm and also the main object of this study. There are 5 loads and 4 lines in Figure 2. We study the monthly energy loss in this network.

Due to data limitations, the collected load data are 1 sampling point per hour. Because load fluctuation is not violent, it is reasonable to consider that the load data are 4 sampling points per hour, but the load data per hour are not changed. To illustrate the error of the original method is large and uncontrollable, two days of the month are selected as a typical day, named day A and day B. The load curves of day A and day B are shown in Figures 3-6.

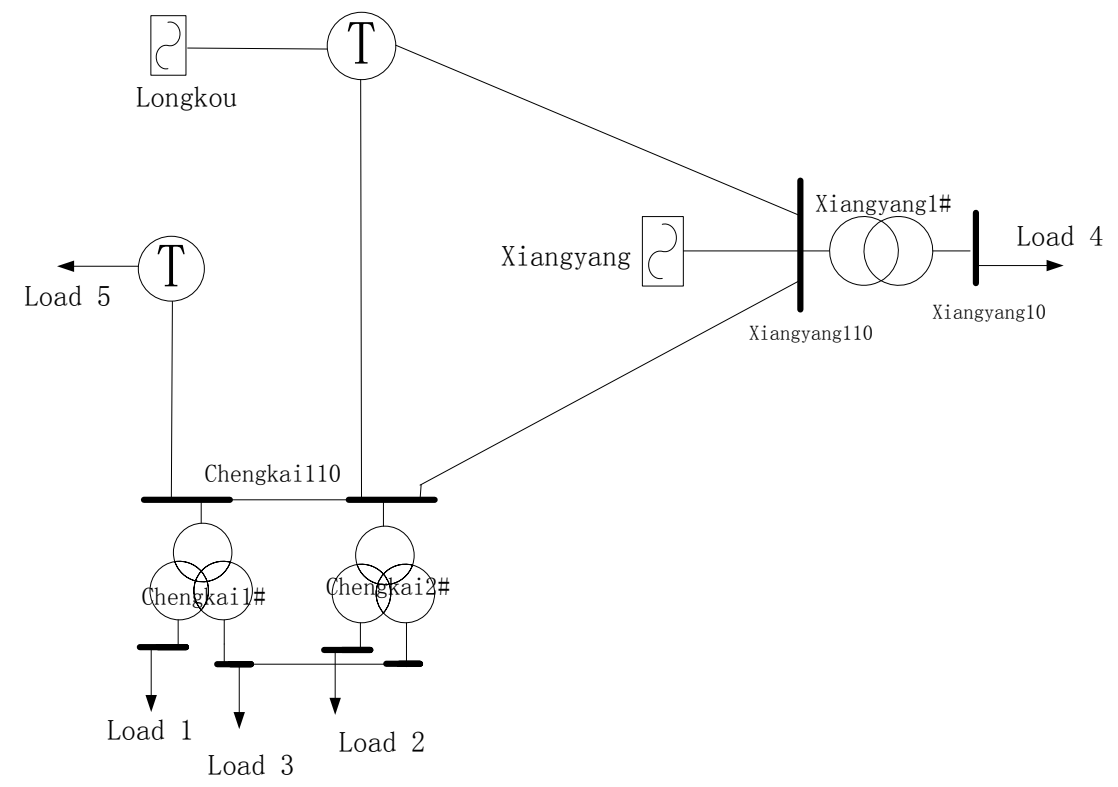

Figure 2. The structure of a $110 \mathrm{kV}$ main network. 


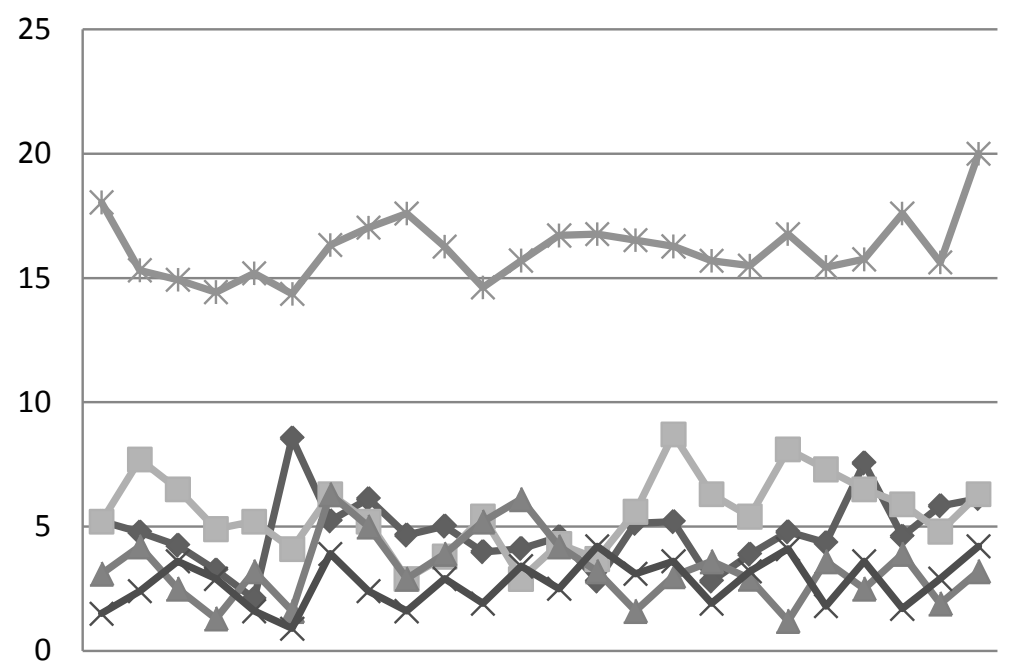

$122 \quad 3 \quad 4 \quad 5 \quad 6 \quad 7 \quad 89101112131415161718192021222324$

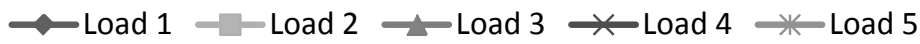

Figure 3. The active load curve of day A.

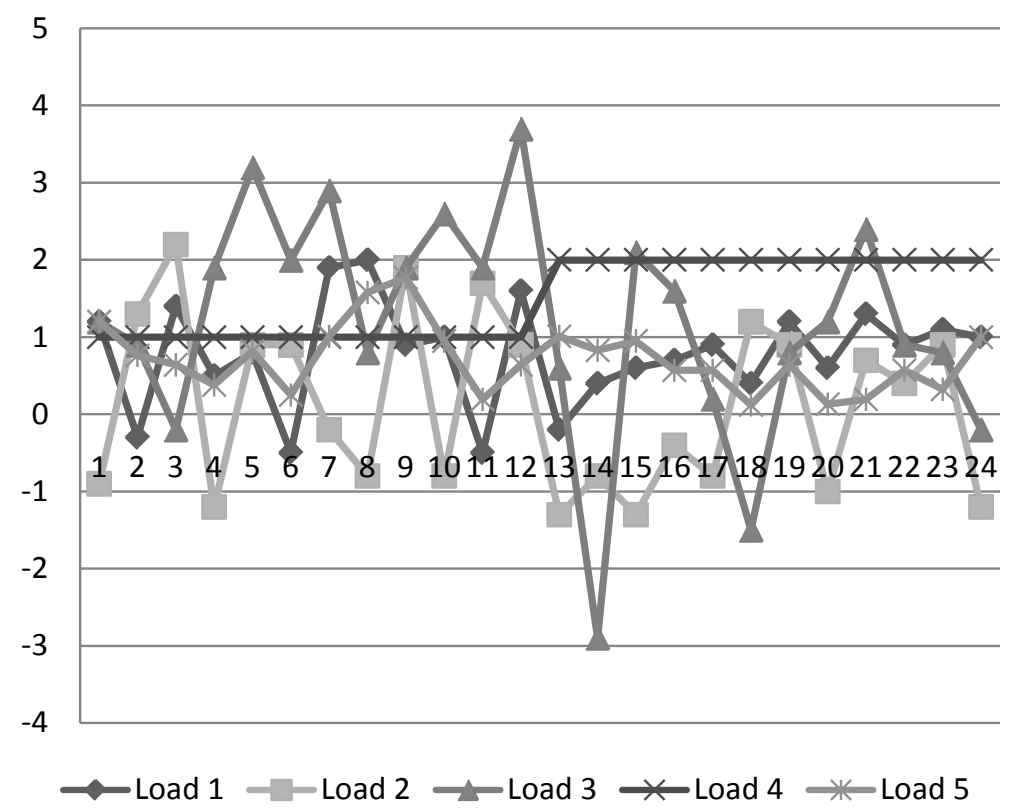

Figure 4. The reactive load curve of day A.

The collected wind power output data of Xiangyang wind farm are 4 sampling points per hour. The power flow is calculated 4 times an hour, namely 96 times a day. The energy loss is accumulated day by day to acquire the monthly energy loss, which is closest to the actual value. The accumulated energy loss value is set to be the reference value, and compared with the result of the original method and the improved method.

The reference value calculated by the power flow is $189.24 \mathrm{MWh}$.

In the improved method, the curve of the conventional power generation and the load is also expressed as the curve of the typical daily expected value. 


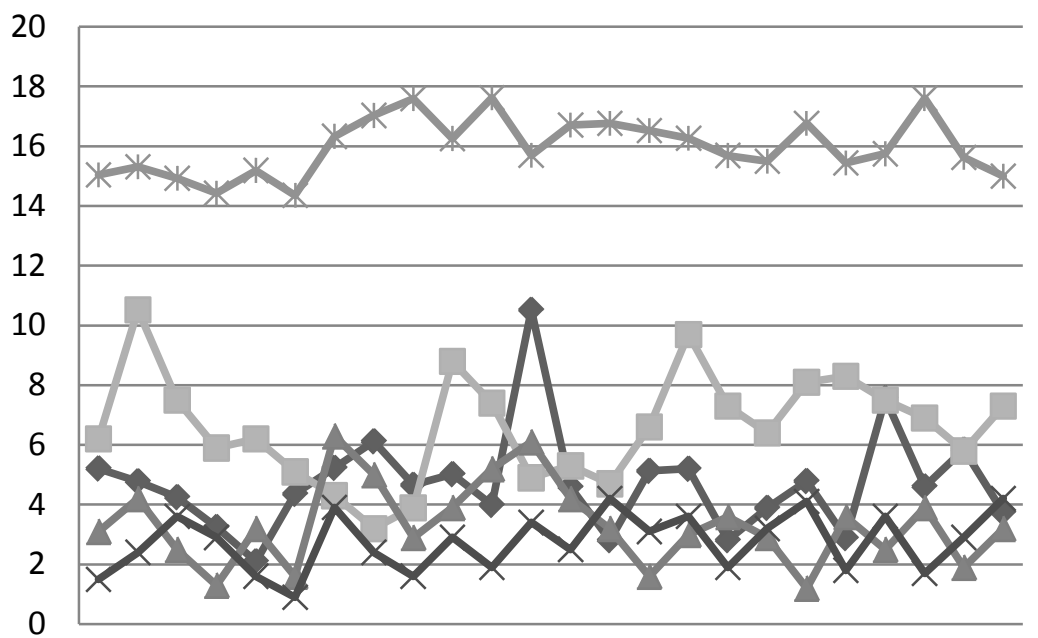

1223445677899101112131415161718192021222324

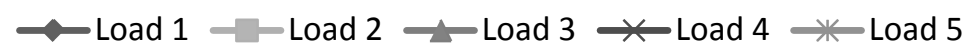

Figure 5. The active load curve of day B.

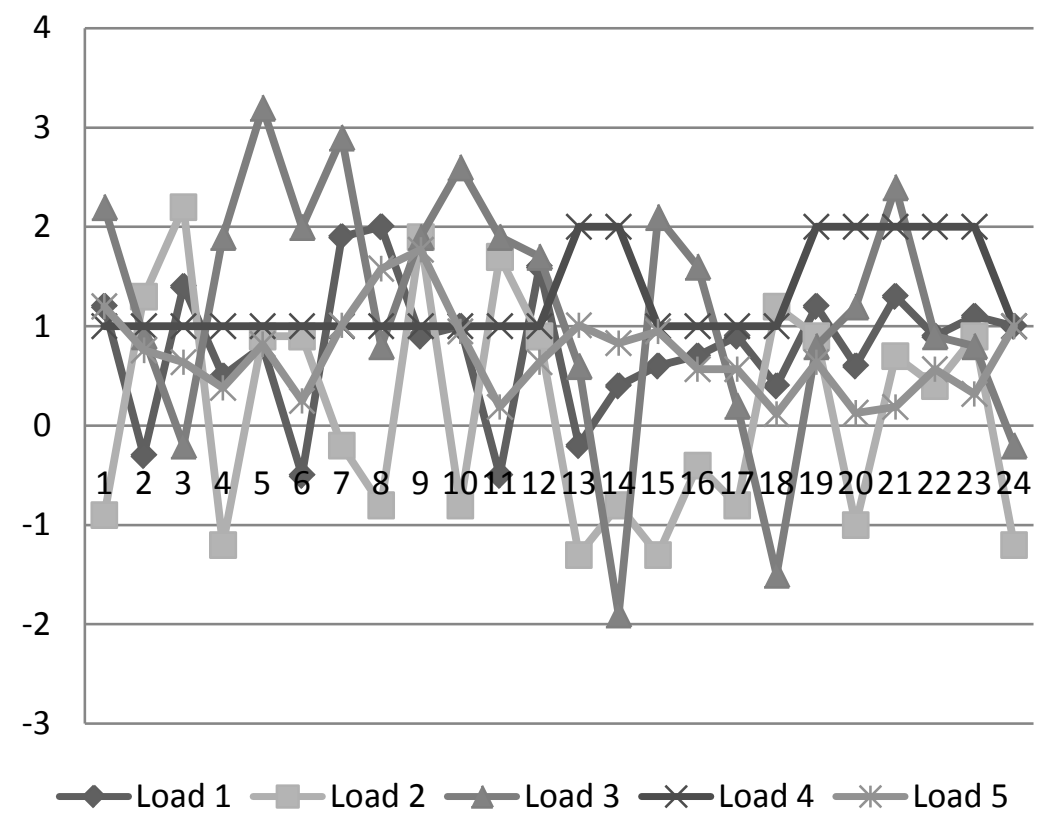

Figure 6. The reactive load curve of day B.

The calculated monthly energy loss in the area is shown in Table 1:

It can be seen from Table 1 that the original method has a large relative error, and the calculated energy loss is different when choosing different typical days, indicating that the error of the original method is large and uncontrollable. When the curve of the typical daily expected output of Xiangyang wind farm is used, the relative error is reduced to $22.99 \%$, and the relative error is reduced to $9.88 \%$ when the curve is corrected to the curve of the typical daily equivalent output of Xiangyang wind farm. It can be shown that the improved method can reduce the calculation error of energy loss. 
Table 1. Results of calculation methods.

\begin{tabular}{ccccc}
\hline \multirow{2}{c}{ calculation methods } & $\begin{array}{c}\text { The calculated } \\
\text { value }(\mathrm{MWh})\end{array}$ & $\begin{array}{c}\text { The reference } \\
\text { value }(\mathrm{MWh})\end{array}$ & the relative error \\
\hline $\begin{array}{c}\text { The original } \\
\text { method }\end{array}$ & dayA & 132.25 & 189.24 & $30.13 \%$ \\
$\begin{array}{c}\text { The improved } \\
\text { method }\end{array}$ & expected output & 127.61 & 189.24 & $32.56 \%$ \\
& equivalent output & 170.56 & 189.24 & $22.99 \%$ \\
\end{tabular}

\section{Conclusion}

In this paper, an improved method of energy loss calculation considering the volatility of the wind power generation is proposed to accurately calculate the monthly energy loss in the power system. Traditionally, repeatedly calculating the power flow for each time periods is undoubtedly an accurate method for the energy loss calculation. However, it suffers from the heavy computational burden. The proposed method in this paper can only calculate the monthly energy loss of the power system via the power flow calculation 24 times, which shows that our method has strong engineering practicability. Additionally, instead of using the typical day's pattern, our method obtains an expected daily curve for selected month, which is also suitable for the case of conventional units and loads. Therefore, the improved method of energy loss calculation for the power system in account of the volatility of wind power generation has very important practical significance.

\section{References}

[1] He,Y.Z. and Wen,Z.Y.(2002) Power System Analysis.HuazhongUniversity of Science and Technology Press,9-15. (in Chinese)

[2] Sun, R.F. (2010) Power Loss Calculation Method for Distribution Network. Northeast Electric Power Technology, 31,34-36.(in Chinese)

[3] Wang, T., Zhang, J.M. and Li, X.P. (2003) Calculation and Evaluation of Scheduled Loss Ratio. Central China Electric Power, 16, 5-7. (in Chinese)

[4] Pierre, P., Henrik, M., Henrik, A., George, N.Papaefthymiou, B.(2008) Kl“ockl. From Probabilistic Forecasts to StatisticalScenarios of Short-term Wind Power Production. Wind Energy, 1-13

[5] Pierre, P., Henrik, A., Nielsen, J.K.,Møller and Henrik, M. and George N. K. (2007) Non-parametric Probabilistic Forecasts of Wind Power- Required Properties and Evaluation. Wind Energy, 10,497-516.https://doi.org/10.1002/we.230

[6] Feng, N. (2008) The Study on Calculation Method of Theoretical Energy Loss in Low-voltage Distribution Network. Xi'an University of Technology, Xi'an.(in Chinese)

[7] Zhang, Y., Wang,J.X. and Wang, X.F.(2014) Review on Probabilistic Forecasting of Wind Power Generation. Renewable and Sustainable Energy Reviews,32, 255-270. https://doi.org/10.1016/j.rser.2014.01.033

[8] Wu, J.S., Ruan, B.,Yu, D.H.,Li, M. and Zhang, B.H. (2015) Distribution Network Energy Loss Calculation Method Considering Wind Power Integration. Preprints of the 5th international conference onElectric Utility Deregulation and Restructuring and Power Technologies.https://doi.org/10.1109/drpt.2015.7432335 
[9] DL/T 686- 1999. Guide of Calculation of Grid Energy Loss.

[10] Q/CSG 1 1301-2008. Technology Standard of Theoretical Calculation of Line Loss.

[11] Zhou,W.J. (2012)Line Loss Analysis of Areas with Wind Power System.Xinjiang University,Xinjiang.

[12] He, J. and Ding, L.N. (2002) Development and Application of the Visual Theoretically Calculating Software for the Distribution Network's Flow Loss. Zhejiang Electric Power, 21, 6-9.

[13] Lu, Y., Zhou, H.G. andLan, C.J. (2013) Analysis of Improved Distribution Network's Line Loss Calculation Method. Electrical Engineering, 1, 98-100.

[14] Liao, X.Q. (2006) Line Loss Calculation Analysis and Loss Reduction Measures of Rural Power Network. Water Power Press.

Submit or recommend next manuscript to SCIRP and we will provide best service for you:

Accepting pre-submission inquiries through Email, Facebook, LinkedIn, Twitter, etc. A wide selection of journals (inclusive of 9 subjects, more than 200 journals)

Providing 24-hour high-quality service

User-friendly online submission system

Fair and swift peer-review system

Efficient typesetting and proofreading procedure

Display of the result of downloads and visits, as well as the number of cited articles

Maximum dissemination of your research work

Submit your manuscript at: http://papersubmission.scirp.org/

Or contact epe@scirp.org 